\title{
Title:
}

\section{Why Internet use? A quantitative examination of the role of everyday life and Internet policy and regulation}

\author{
Author: \\ Dr. Panayiota Tsatsou
}

\section{Affiliation:}

Swansea University, UK

\begin{abstract}
Regardless of the technological advances achieved so far and the often techno-deterministic approaches to the information society, inequalities in the access to, distribution and use of ICTs such as the Internet still highlight the importance of digital divides. The complex nature and continuing importance of the unequal rates of use of ICTs such as the Internet invite research to examine their drivers. Ordinary people's everyday life and their awareness and evaluation of policy and regulation are influential factors in how Internet use takes shape today. This is the argument made by this article and supported by a survey of 1,001 Internet users and non-users in Greece. Particular attention is paid to how aspects of everyday life and culture, such as resistance to ICTs, and people's awareness and evaluation of Internet policy and regulation in areas such as online security and privacy can explain Internet use.
\end{abstract}

Keywords: Internet use, everyday life, resistance, policy, regulation. 


\section{Introduction}

Level and breadth of Internet use are a core element of the complex, problematic and rapidly changing phenomenon of digital divides [1]. Gunkel argues that 'the problems of the digital divide have been and probably will continue to be moving targets', suggesting that 'the term's definition should be similarly mobile' [2, p. 505]. Some others even wonder whether the term has any real meaning $[3 ; 4]$.

Researchers' attention has conventionally been drawn to socio-economic and demographic differences as the main source of divisions between 'haves' and 'have-nots' of Information and communication Technologies (ICTs) such as the Internet. Today, however, there is an increasing volume of literature that challenges the simplistic 'bipolar societal split' between 'haves' and 'havenots' and illustrates the role of physical, digital, human and social resources in posing barriers to access to and use of ICTs such as the Internet [5, p. 6]. Regarding the aspects of the divides which are of importance today, it is argued that, in relation to the Internet, although 'social divisions in internet access continue to exist' [6, p. 29], the breadth of Internet activities, abilities, skills and means to overcome potential barriers to functional Internet use, as well as 'techno-culture' [7], are becoming increasingly important aspects of digital divides. Thus, inequalities in skills for and usage of ICTs such as the Internet [8, p. 324; 9, p. 7], as well as in 'cohort' and 'awareness' [10, p. 35-65], are now seen as additional divides.

Lately, intellectually advanced approaches to digital divides speak about the importance of 'information habitus' and how various qualities and disparities in everyday life influence the quality of Internet access, usage and skills [11]. Others speak about the role of culture (social or national) in the usage of ICTs such as mobile phones [12], contributing further to the effort of the research to move the discussion beyond the role of economics and socio-demographics in digital inequalities. On the other hand, there is not much research that provides empirical insights directly into whether people's evaluations and awareness of policy and regulation schemes that strive against the various 
aspects of digital divides actually influence ICT and Internet related inequalities.

This article focuses on divisions related to Internet usage, with the latter being measured by Internet use and quality-of-use parameters, such as frequency of use, online activities and type of Internet connection. Taking into consideration insights and gaps in the literature, the article argues that Internet use is inseparable from ordinary people's everyday life and culture and heavily dependent on Internet policies and regulations as these are evaluated and perceived by ordinary people. Thus, the article pursues the question of 'how Internet use can be explained if taking into account the role of people's everyday lives, on the one hand, and their evaluation and awareness of Internet policies and regulations, on the other?' It reports on a nationally representative survey of 1,001 Internet users and non-users in Greece and employs multivariate modelling to test, among other commonsense parameters, the role of indicators related to ordinary people's everyday life, on the one hand, and their awareness and evaluation of Internet policies and regulation on issues of importance, such as online privacy and security, on the other.

\section{Why everyday life and Internet policy/regulation?}

Everyday life is considered highly interconnected with people's experiences of technology, allowing the phenomenon of digital divides to be seen in association with socio-cultural contexts of life $[13$, p.5; $11 ; 12]$.

'Domestication' $[14 ; 15 ; 16]$ and the argument that the study of ICTs does not end with people's decisions to buy them, since technology is domesticated through 'taming of the wild and a cultivation of the tame' [17, p. 223], hold a certain research value in this respect. Also, Schutz's 'lifeworld' makes an important contribution to the study of technology in an everyday life framework. In the examination of how people experience their 'everyday life-world' [18, p. 3], the 
agent is viewed as manipulating physical and social structures in the pursuit of his/her purposes of living. Schutz's 'lifeworld' points out the interplay between imposed and freely chosen actions and choices in everyday life [18, p. 100], locating the individual in the social and cultural environment and defining 'the province of what is open to me now to control' [18, p. 111]. On the other hand, the concept of 'domestication' implies that the 'domestic' is to be looked at independently and not in any extensive dialectic with outside or divergent environments where ICTs are appropriated or dismissed. In any case, these conceptual tools can be of use for the examination of how ICTs and the Internet in particular are adopted on the grounds of people's freely made decisions and of the possible constraints placed by the systemic contents of everyday life.

Although in this article I provide a far from exhaustive account of everyday life, I attempt to operationalise the deeply qualitative and often broadly defined concepts of everyday life, domestication and 'lifeworld', and to transform these into measurable and reliable quantitative indicators for the prediction of Internet use. Thus, this article highlights certain aspects of everyday life and their role in people's decisions to use the Internet by capturing the way people position the Internet in everyday life in general (e.g. the Internet as a necessary tool for everyday life) and in specific domains of the everyday (e.g. workplace, social life, daily routines etc).

Particularly parameters of social engagement, consent or resistance to technology are all important elements of everyday life and culture and constitute a key aspect of the everyday to consider when studying people's decision to use or not ICTs such as the Internet. Historians of technology argue that 'the resistance to innovation is identified as a central element governing the success of new inventions' [19, p. 325]. Also, a good number of scholarly works have reported on resistance to technology $[20 ; 21 ; 22 ; 23)$, mostly looking at particular parts of the population (e.g. children) and specific areas of everyday life (e.g. the workplace). Bauer's work [24, p. 13-5] examines resistance to ICTs in general and for the whole population, questioning the idea of 'resistance' as a diversion from the 'one best way'. He identifies intrinsic qualities and dynamics of resistance in the broader 
context of the everyday, classifying resistance as 'active or passive', 'individual or collective', and as referring to 'technology design', 'technology effects' or 'technology governance' [24, p. 16-21]. With regard to the Internet, Bauer considers resistance a useful everyday life based attitude to the Internet that signals the mismatch of expectations between Internet users and designers [25, p. 113].

In this article I do not enter into normative judgements of resistance to the Internet, as I aim to illustrate that this aspect of everyday life and culture can be a useful analytical tool for disentangling the drivers of Internet use. Thus, through multivariate statistical analysis, this article explores, for instance, people's views of whether the Internet jeopardises moral values and traditions and the role of such views in people's decision to use the Internet or not. Other empirical studies have touched upon people's resistance to or dismissal of the Internet in particular, ${ }^{i}$ but this article presents a more complex study which combines the resistant element of everyday life with general everyday life routines as well as particular domains of the everyday, thus providing insights into the joint role of the multiple facets of everyday life in Internet use.

Nevertheless, the scope of this article goes beyond everyday life. It goes beyond arguments that the meaning and relevance of ICTs such as the Internet are shaped by identities in the everyday lives of different groups [26, p. 198] and that 'lifestyle' is strongly correlated to use of and interest in new technologies [27]. The latest trends in everyday life studies attempt to extend the scope of this research into the field of politics and argue that people's place in society has a role to play not only in their attitudes to and evaluation of technology, but also in politics. In this sense, they argue that everyday life entails 'important implications for public policies and strategy... which may serve to challenge or enhance the kinds of thinking and considerations that currently inform policy decisionmaking or practices' $[28$, p. 3-4].

From this perspective, the notion of 'social shaping' concerns not only technology but also decision-making, with different everyday life contexts influencing the development of different perceptions and evaluations of policy models. From this point of view, the European Media 
Technologies in Everyday Life Network (EMTEL) explored the links between the everyday and ICTs and the importance of those links for policy-making, aiming to provide the EU authorities with an insight into the possible insufficiencies of ICT policy frameworks in Europe:

...without this sensitized investigation of the dynamics of the everyday and of innovation as a contested process of social as well as technological change we will misread and misunderstand the realities of innovation and the implications of those realities for policy [13, p. 8-9].

Drawing upon the literature looking at the linkages between everyday life and policy in the information society, this article examines a second set of factors in Internet use, those related to people's evaluation and awareness of Internet policy and regulation. Currently, not much empirical research exists on the integration of Internet technologies in specific everyday life settings and in connection with the political and regulatory domains of activity. This is a gap, as technology is an area in which people's everyday life and culture and their perceptions of decision-making matter, entailing implications for the way in which technology is diffused as well as for the actual effectiveness of policy and regulatory practices in dealing with issues of importance for people's use of technologies like the Internet. Thus, more attention should be paid to the reciprocal and highly interdependent relationship between technology, people's everyday life settings and policymaking when raising questions about the drivers of divisions in Internet use.

At the same time, the study embraces more conventional indicators and factors in Internet use, such as demographics, Internet access and other media use factors, so as to place Internet use within a broader system where other parameters operate and more conventional accounts of use and related inequalities maybe reviewed. Finally, the study reported here examines Internet use in Greece, but the article aspires to move beyond national particularities, raising the potential applicability of this study to other case studies and to broader research literature in the field. 
Hence, in pursuing the research question of 'how Internet use can be explained if taking into account the role of people's everyday lives, on the one hand, and their evaluation and awareness of Internet policies and regulations, on the other?', the article operationalises and quantitatively examines the key concepts briefly presented in this section, while it tests the following hypothesises:

a. first, ordinary people's everyday life and possible instances of resistance to technology in the context of the everyday are strong predictors of Internet use;

b. second, ordinary people's evaluation and awareness of Internet policy and regulation (i.e. policies, regulations and related authorities concerning issues of online privacy and security) are strong predictors of Internet use;

c. third, indicators conventionally used in explaining Internet use, ${ }^{\text {ii }}$ such as demographics and Internet access/other media use, are only marginally important predictors.

These hypotheses are tested and an answer to the research question is provided in the empirical part of the article. The next section presents the survey design and the analytical methods applicable to the task at hand.

\section{Method}

The empirical insights reported in this article draw upon the data collected in a telephone survey (CATI) of a representative sample of 1,001 Internet users and non-users in Greece. The survey sample and the measures (factors) used in the analysis of the survey data are explained below.

\subsection{Survey design}

The survey design was based on the research objectives, theoretical framework and research hypotheses employed to explore the main forces influencing Internet use. The survey traced 
behaviour patterns, measurable opinions and attitudes to the Internet in the context of people's everyday life, while shedding light on social awareness and the evaluation of policies and regulation in the field.

Systematic sampling was applied in order a representative sample of respondents to be drawn from Attica (i.e. the urban region where about half of the population of Greece resides). Systematic sampling is easily adjustable to local and regional samples [29, p. 87] and ensures the same precision as random sampling, while being less laborious and better organized. Weighting was applied in order to remove demographic biases such as the over-coverage of females, the participation of a large number of people aged 25-39 and that very few people aged $65+$ responded. The sample had the following characteristics: males \& females, aged 15-85 years, and permanent residents of Attica.

The survey data were recorded and converted into an analysable format through the usage of CATI software (IT by DESAN). They were analysed quantitatively with SPSS software, employed on the basis of careful consideration of the structure and types of questions addressed in the questionnaire and in accordance with the study's aims.

\subsection{Measures}

The role of the predictors of everyday life and Internet policy and regulation in Internet use was measured both separately and jointly. At the same time, the analysis contextualised everyday life and policy parameters, also accounting for the role of conventional drivers of digital divides, such as access to Internet technology, other media use and demographics.

The predicted (dependent) variable of Internet use was captured by the following measures (see Table 1):

- Internet use: a dichotomous variable $(1=$ use, $2=$ non-use, Internet use $=44.5 \%)$. 
- Quality of Internet use > an aggregate measure of the following three variables:

○ Frequency of use, consisting of a 7-point scale from 1 ('several times a day') to 7 ('less often') $(\mathrm{M}=2.08, \mathrm{SD}=1.264)$.

○ Internet connection, consisting of five dummies from 1 ('Dial-up') to 5 ('Wireless'). In the multivariate analysis it was operationalised as a dichotomous measure $(1=$ dial-up, $0=$ otherwise $)(\mathrm{M}=0.4596, \mathrm{SD}=0.49904)$.

- Online activities, consisting of one dummy per activity. In the multivariate analysis this was operationalised as the number of online activities users engage in when going online $(\mathrm{M}=$ 2.4261, $\mathrm{SD}=1.28106)$

As regards the predictors of Internet use, everyday life parameters and the way in which people position the Internet in the everyday were captured by the following variables (see Table 1):

- Everyday life and the Internet (all). This is a construct that measures the positioning of the Internet in everyday life as well as the possible existence of resistant attitudes to the Internet in the context of the everyday. It consists of the following eight variables (5- and 4-point scale), all standardised so that the higher the value the more positive the scale $(\mathrm{M}=3.3189, \mathrm{SD}=0.44307$, $\left.\mathrm{a}=0.88^{\mathrm{iii}}\right)$ :

- The Internet as a positive tool in everyday life. Here the respondents indicated on a 5 point scale from 1 ('strongly disagree') to 5 ('strongly agree') whether the Internet is a technology that positively changes our lives $(\mathrm{M}=3.72, \mathrm{SD}=1.027)$.

- The Internet as a necessary tool in everyday life. Here the respondents indicated on a 5 point scale from 1 ('strongly disagree') to 5 ('strongly agree') whether the Internet is a necessary tool in everyday life $(\mathrm{M}=3.36, \mathrm{SD}=1.120)$.

- The Internet as a danger for security. Here the respondents indicated on a 5 point scale from 1 ('strongly disagree') to 5 ('strongly agree') whether the Internet is a danger to users' security in terms of online fraud and privacy violation $(\mathrm{M}=3.89, \mathrm{SD}=1.024)$.

- The Internet as a danger for social life. Here the respondents indicated on a 5 point scale from 
1 ('strongly disagree') to 5 ('strongly agree') whether the Internet is a danger to users' social life and offline relationships $(\mathrm{M}=3.37, \mathrm{SD}=1.174)$.

- The Internet as replacing the individual in the workplace. Here the respondents indicated on a 5 point scale from 1 ('strongly disagree') to 5 ('strongly agree') whether the Internet replaces the individual worker in the workplace $(\mathrm{M}=2.99, \mathrm{SD}=1.211)$.

- The Internet as jeopardising values and traditions. Here the respondents indicated on a 5 point scale from 1 ('strongly disagree') to 5 ('strongly agree') whether the Internet can jeopardise people's moral values and traditions $(\mathrm{M}=3.35, \mathrm{SD}=1.218)$.

- The Internet in communication. Here the respondents indicated on a 5 point scale from 1 ('very negatively') to 5 ('very positively') how the Internet affects the ways people communicate with one another in everyday life $(\mathrm{M}=2.81, \mathrm{SD}=1.057)$.

- The Internet in daily routines. Here the respondents indicated on a 4 point scale from 1 ('no role at all') to 4 ('a major role') how much of a role the Internet plays in the way people go about their daily routines and activities $(\mathrm{M}=3.06, \mathrm{SD}=0.805)$.

- Everyday life and the Internet (users). This measures on a 4 point scale from 1 ('not at all') to 4 ('a lot') users' views on whether future deprivation of the Internet would affect their daily routines and activities $(\mathrm{M}=2.63, \mathrm{SD}=1.048)$.

The predictor of Internet policy and regulation was captured by measuring people's evaluation and awareness of Internet policy and regulation (see Table 1):

- Evaluation of Internet policy and regulation (all). ${ }^{\text {iv }}$ This is a construct that measures on a 5 point scale from 1 ('strongly disagree') to 5 ('strongly agree') the mean score of people's evaluation of the effectiveness of national and EU policies and regulation in coping with online security and privacy risks $(\mathrm{M}=2.8987, \mathrm{SD}=0.82493, \mathrm{a}=0.92)$. It consists of the following four variables:

- Evaluation of national laws and policies on security risks. Here the respondents evaluated how national laws and policies can cope with online security risks $(\mathrm{M}=2.66, \mathrm{SD}=1.051)$. - Evaluation of national laws and policies on privacy risks. Here the respondents evaluated 
how national laws and policies can cope with online privacy risks $(\mathrm{M}=2.79, \mathrm{SD}=1120)$.

- Evaluation of EU laws and policies on security risks. Here the respondents evaluated how EU laws and policies can cope with online security risks $(\mathrm{M}=3.08, \mathrm{SD}=1.074)$.

- Evaluation of EU laws and policies on privacy risks. Here the respondents evaluated how EU laws and policies can cope with online privacy risks $(\mathrm{M}=3.14, \mathrm{SD}=1.050)$.

- Evaluation of national policies and regulation (users). This is also a construct which measures on a 5 point scale from 1 ('very dissatisfied') to 5 ('very satisfied') the mean score of Internet users' satisfaction with the way in which national policies and regulation protect users' security and privacy on the Internet $(\mathrm{M}=2.6291, \mathrm{SD}=0.87989, \mathrm{a}=0.84)$. It consists of the following two variables:

○ Internet users' satisfaction with national laws and policies on security risks. Here the users stated their satisfaction with how national laws and policies protect users' online security (M $=2.67, \mathrm{SD}=0.924)$

- Internet users' satisfaction with national laws and policies on privacy risks. Here the users stated their satisfaction with how national laws and policies protect users' online privacy $(\mathrm{M}=$ 2.61, $\mathrm{SD}=0.969)$.

- Awareness of Internet policy and regulation. This measures on a 5 point scale from 1 ('strongly disagree') to 5 ('strongly agree') Internet users and non-users' evaluation of how much people in general are aware of Internet policies and regulation $(\mathrm{M}=3.08, \mathrm{SD}=1.014)$.

- Awareness of Internet authorities (all). This is a dichotomous variable ( 1 = 'heard', 2 = 'not heard') that measures people's awareness of authorities that monitor the application of Internet laws and policies in the country $(\mathrm{M}=1.75, \mathrm{SD}=0.436)$.

- Awareness of Internet authorities (users). This is a construct that measures on a 3 point scale (1 $=$ 'yes', 2 = 'no', 3 = 'not sure/it depends') the mean score of users' awareness of authorities to which they might address themselves if facing problems online $(\mathrm{M}=1.8357, \mathrm{SD}=0.37377, \mathrm{a}=$ $0.75)$. It consists of the following variables: 
- Users' awareness of Internet authorities if encountering security problems. The users stated whether they are aware or not of authorities if they face security problems online $(M=1.80$, $\mathrm{SD}=0.397)$

- Users' awareness of Internet authorities if encountering privacy problems. The users stated whether they are aware or not of authorities if they face privacy problems online $(\mathrm{M}=1.80$, $\mathrm{SD}=0.398)$

- Users' awareness of Internet authorities if encountering difficulty in use. The users stated whether they are aware or not of authorities if they face problems in using the Internet $(\mathrm{M}=$ $1.73, \mathrm{SD}=0.446)$

- Perceived accountability of Internet authorities. This is a construct that measures on a 5 point scale from 1 ('strongly disagree') to 5 ('strongly agree') the mean score of people's views of the social accountability of national and EU Internet authorities $(\mathrm{M}=3.1010, \mathrm{SD}=0.87588, \mathrm{a}=$ 0.81). It consists of the following variables:

○ National authorities' accountability. Here the respondents stated whether national authorities fail to take the citizen's voice on the Internet into account $(\mathrm{M}=3.19, \mathrm{SD}=1.092)$.

- EU authorities' accountability. Here the respondents stated whether EU authorities fail to take the citizen's voice on the Internet into account $(\mathrm{M}=3.08, \mathrm{SD}=1.014)$.

Lastly, the analysis included predictors which are commonly considered when exploring key aspects of digital divides such as Internet use:

- Access indicators: Internet availability $(\mathrm{M}=2.66, \mathrm{SD}=2.982)$ and computer availability $(\mathrm{M}=$ $3.24, \mathrm{SD}=2.390)$

- Other media use: computer use ( 1 = 'yes', 2 = 'no'; $\mathrm{M}=1.45, \mathrm{SD}=0.498)$; frequency of reading newspapers from 1 ('several times a day') to 9 ('don't read newspapers') $(\mathrm{M}=5.26, \mathrm{SD}=$ 8.621); and frequency of watching $T V$ from 1 ('several times a day') to 10 ('not at all') ( $\mathrm{M}=$ $1.85, \mathrm{SD}=3.121)$

- Patterns of Internet use: history of Internet use from 1 ('last six months') to 4 ('more than three 
years ago') $(\mathrm{M}=3.40, \mathrm{SD}=0.879)$.

- Demographics: ${ }^{\mathrm{v}}$ age $\mathrm{vi}(\mathrm{M}=28.76, \mathrm{SD}=17.785)$, gender $(1=$ 'male', 2 = 'female'; $\mathrm{M}=1.51, \mathrm{SD}$ $=0.500)$, education (from $1=$ 'none or primary' to $7=$ 'postgraduate') $(\mathrm{M}=5.18, \mathrm{SD}=11.522)$, and children in the household $(\mathrm{M}=3.16, \mathrm{SD}=12.805)$.

-----Table 1 about here -----

\subsection{Modelling}

Multivariate modelling was employed in order to test the role of everyday life and people's evaluation and awareness of Internet policy and regulation in Internet use, and to control for interrelations between these through a partial correlation analysis. More specifically:

- Multiple linear regression. This was employed in the analysis when the response variable was a non-dichotomous, continuous, interval-level variable (e.g. frequency of Internet use). Because more than one explanatory variable is used at once, this technique has the advantage that even tentative claims that the effect of a variable on the response variable is causal can be put forward only if this effect survives conditioning on all control variables which are thought of as relevant [for more, see 30]. An F test was used to assess whether all the coefficients of the independent variables are jointly equal to zero, and T-test statistics were used to assess the significance of each independent variable. Further, the R-squared statistic accounts for the strength of each regression model.

- Logistic regression. This was used when the dependent variable was observed and dichotomous (e.g. Internet use). As with multiple linear regression, a logistic regression involves modelling differences between individuals, using multiple explanatory variables. The explanatory variables can be of any level of measurement and are used in exactly the same way as in a multiple regression [for more, see 30]. Omnibus chi-square tests were used to assess whether all the independent variables are jointly significant. Individual chi-square tests were used to assess 
the significance of each explanatory variable and Nagelkerke $\mathrm{R}^{2}$ to account for the strength of the model.

In all regressions the ideal model was identified through Backward Wald that allows all selected predictors to be included in an initially saturated model and then eliminates all variables not significantly contributing to the model.

\section{Results}

This section presents the results of multivariate modelling and how these results tested the three hypotheses of the study (see p. 8):

\subsection{Internet use}

In order to assess the factors that influence Internet use in itself (not qualitative aspects of use), logistic regression was employed. The factors included in the model were selected on the grounds of what the literature reports about the drivers of Internet use (e.g. Internet access, patterns of media use, computer use, demographics, etc). Also, the study tested the particular role of people's everyday life (i.e. everyday life and the Internet (all)) and their evaluation and awareness of Internet policy and regulation (i.e. evaluation of Internet policy and regulation (all), perceived accountability of Internet authorities, awareness of Internet policy and regulation, and awareness of Internet authorities (all)).

Table 2 presents the results of the last step (step 10) of the Backward Wald:

-----Table 2 about here -----

As the table reveals, the Omnibus Chi-square test $\left(\mathrm{x}^{2}=340.264, p<.001\right)$ supports the explanatory strength of the model depicted in the table. This is also supported by Nagelkerke $\mathrm{R}^{2}(0.789)$ which is 
close to 1 , showing that a significant proportion of variation of the dependent variable is explained by this model. At the 0.05 level of significance, people aged between 15 and 39 years old, those with a higher education, and men are significantly more likely to use the Internet than older people, those with a lower education, and women, respectively. Also, individuals who have Internet access at home and those who are computer users are significantly more likely to be Internet users than those with no home access to the Internet and those who do not use a computer, respectively.

On the other hand, there is insufficient evidence that Internet use depends directly on other media use (e.g. frequency of reading papers/watching TV), on the way in which the Internet is positioned in people's everyday lives $(B=-0.599, \mathrm{p}>0.10)$, on people's evaluation of Internet policy and regulation $(\mathrm{B}=-0.215, \mathrm{p}>0.10)$ or on other policy and regulatory factors. Although these results reject the first and second hypothesis and do not strongly support the third hypothesis of the study, all the hypotheses are tested below for quality aspects of Internet use.

\subsection{Quality of use}

Quality of use is measured by the variables of frequency of use, online activities and type of Internet connection, meaning that high frequency of use, a large number of activities and broadband Internet connection suggest high quality of Internet use. The data on quality of use were only available for Internet users, thus the regressions below were limited to those who said they were users.

\subsubsection{Frequency of use}

The factors that influence frequency of Internet use were assessed through a multiple linear regression. The analytical strategy was to include in the initial model all possible factors regarded by the research literature as associated with frequency of use (e.g. history of use, Internet connection, Internet availability, demographics, etc). Variables that measure users' positioning of 
the Internet into their everyday lives, as well as their evaluations and awareness of policy and regulation on Internet privacy and security matters were also included ${ }^{\text {vii }}$.

The results of the last step (step 9) of the Backward Wald are shown in Table 3:

-----Table 3 about here -----

The R-Squared of the model is 0.391 , implying that the independent variables explain 39.1 per cent of the variability in frequency of use. Thus, the null hypothesis of no joint effect of the independent variables on frequency of use was rejected $(\mathrm{F}=15.299 p<0.001)$, supporting the explanatory strength of the model. At the 0.05 level of significance, the results in Table 3 show that, in terms of demographics, users who have children in their household make significantly less frequent use of the Internet. Also, users with Internet at home tend to be more frequent users than those without an Internet connection, whereas those with a dial-up Internet connection at home are less frequent users than those with broadband. In addition, users who have used the Internet for a longer time also use it more frequently than those who have been using the Internet for a shorter time. In terms of the role of everyday life parameters, users who have a stronger perception that not accessing the Internet would affect their daily routines tend to make significantly more frequent use of the Internet. Finally, in terms of policy and regulation, users who are aware of Internet policy and regulatory authorities they can contact in case of difficulty with Internet use, security or privacy risks on the Internet are also significantly more frequent users than those lacking awareness of such authorities.

Thus, access to the Internet, patterns of Internet use (e.g. history and Internet connection), certain aspects of positioning of the Internet in users' everyday lives and users' awareness of Internet policy and regulatory authorities influence frequency of Internet use, confirming the first and partly the second hypothesis of the study. As regards the second hypothesis in particular, frequency of use does not depend on users' evaluation $(B=-0.092, p>0.10)$ and awareness $(B=-0.064, p>0.10)$ of Internet policy and regulation, or on the perceived accountability of Internet authorities $(B=-0.049$, 
$p>0.10)$. Also, it is important to note that the demographics of age $(B=-0.050, p>0.10)$, gender $(B=0.026, p>0.10)$ and education $(B=-0.068, p>0.10)$ do not influence frequency of use, a finding that supports to an extent the third hypothesis and challenges, for instance, the idea that young users are more frequent users than middle-aged and older people.

\subsubsection{Online activities}

A multiple linear regression was conducted in order to assess the factors that affect users' online activities. The independent variables were the same as for the 'frequency of use' model and their selection relied on the same rationale as above. Results of the last step (step 19) of the Backward Wald are presented in Table 4:

-----Table 4 about here -----

The R-Squared (0.132) implies that variability in the type of Internet connection and in users' positioning of the Internet in everyday life accounts for 13.2 per cent of the variability in the number of users' online activities. Although this model does not explain a particularly large proportion of the variance of online activities, the null hypothesis of no joint effect of the independent variables on the number of online activities is rejected $(\mathrm{F}=10.577, p<0.001)$. More specifically, at the 0.05 level, users with a fast Internet connection and those who perceive Internet use as important for their everyday lives tend to engage in a significantly larger number of online activities, thus confirming the first hypothesis. On the other hand, no evidence was found that evaluation $(B=0.013, p>0.10)$ and awareness $(B=0.138, p>0.10)$ of Internet policy and regulation, awareness of Internet authorities $(B=0.019, p>0.10)$ or perception of the accountability of authorities $(B=-0.167, p>0.10)$ have a role to play in the breadth of their Internet usage. Likewise, users' particular evaluation of Internet policies and regulation $(B=0.082$, $p>0.50)$ and awareness of Internet authorities $(B=-0.007, p>0.10)$ do not matter, thus challenging the second hypothesis of the study. Also, socio-demographics do not have any 
influence at all, which supports to an extent the third hypothesis and challenges in turn commonsense perceptions of the role of education and age in breadth of Internet usage.

\subsubsection{Internet connection}

Research has shown that the type of Internet connection influences the range of online activities and the quality of the experience of Internet usage overall.

In order to assess the factors that influence the likelihood of the Internet user accessing the Internet through dial-up rather than broadband, a logistic regression was conducted. Factors broadly considered to influence the type of Internet connection in the household were included in the model (e.g. demographics, frequency and history of use, etc). Also, the indicator of online activities was included in the context of the hypothesis that those who engage in more activities are inclined to obtain a faster Internet connection, while, as noted above, broadband might in turn drive people to engage in a wider range of activities. Lastly, factors concerning the positioning of the Internet in everyday life, as well as the user's evaluation and awareness of Internet policy and regulation were included.

After 18 reiterations, the model (Table 5) below was selected as the best fit with the data:

-----Table 5 about here -----

The statistic of Nagelkerke $\mathrm{R}^{2}=0.235$ indicates that this model does not explain a particularly large proportion of the variance of the predicted variable of Internet connection, somehow challenging the strength of the model. On the other hand, the Omnibus Chi-square test $\left(\mathrm{x}^{2}=28.922, p<.001\right)$ rejects the null hypothesis that none of the included independent variables has an effect on Internet connection $(p<0.001)$. Thus, at the 0.05 level, the results show that users who make more frequent use of the Internet and those who carry out a greater number of online activities are significantly less likely to have a dial-up connection. On the other hand, none of the other independent variables 
has a significant effect on the likelihood of having a dial-up connection, thus rejecting the first and second hypotheses of the study, while supporting to an extend the third hypothesis.

Overall, the above three models found that frequency of use and online activities are affected by users' positioning of the Internet in everyday life, thus supporting the first hypothesis of the study. Also, they found some narrow effect of users' approach to Internet policy and regulation (i.e. users' awareness of Internet authorities) on frequency of use, thus offering limited support to the second hypothesis. On the other hand, demographics, access indicators and other commonsense factors appear less strong predictors of quality of use than use per se, thus confirming to an extent the third hypothesis. Lastly, the various aspects of quality of Internet use appear to be interlinked, with the number of online activities being influenced by the existence of dial-up connection at home and with the latter being influenced by the frequency of use and number of online activities.

\section{Discussion}

The above multivariate analysis informs research and theory and paves the way for reaching useful conclusions about digital divides in general and Internet use in particular.

The above results confirm the first and the second hypotheses, as they illustrate that the way in which the Internet is positioned in everyday life and users' awareness of Internet authorities are significant factors influencing quality parameters of Internet use, but not use in itself. Also, the analysis has found that socio-demographics and other commonsense factors (e.g. Internet access, media use, etc) are still important predictors of Internet use, but not equally important for quality of use, thus giving only some support to the third hypothesis. 
These conclusions may only partly confirm the three hypotheses, but they do not challenge the overall conceptualization and research rationale of the study. As regards the research question, they indicate the importance of people's everyday lives and the way people approach Internet policy and regulation for qualitative aspects of Internet use, while showing the complex and relative role of these parameters in digital divides in general. Also, the means and standard deviations of the measures provided in the Method section indicate quite a complex and interesting picture of everyday life and culture and policy/regulation in relation to the Internet. The majority of the respondents are non-users, while most users are frequent users but with limited breadth of usage and a significant percentage of dial-up connections to the Internet. People seem to have quite a puzzling understanding of the role of the Internet in everyday life, as they evaluate positively the positioning of the Internet in daily life, while regarding the Internet as causing risks in particular domains of the everyday (e.g. social life, security, privacy, etc). Also, they seem to have low appreciation of the effectiveness and social accountability of Internet policies and regulation in general, but far lower for national than EU policies and laws. At the same time, the multivariate analysis problematised the role of conventional socio-demographic and access parameters, especially as far as qualitative aspects of divides are concerned (e.g. quality of Internet use).

In light of these findings, the article does not argue for the clear-cut and linear role of people's everyday life and their evaluation and awareness of Internet policy and regulation in Internet use, but it offers useful insights into how everyday life and policy parameters can potentially be used in empirical research on the issue at stake. As regards the role of everyday life, for instance, my findings go beyond recent attempts of research to view digital divides in a socio-cultural context. For instance, Baron and af Segerstad's (2010) exploration of the role of culture in mobile phone usage in an international context (USA, Korea, Japan, Sweden and Italy) does not specify the aspects of culture which are of interest, it lacks a ground where arguments related to national cultures can be based (e.g. how do we know social culture in the USA, in Japan, etc?), and it presents culture as a parameter the role of which can be examined in isolation from other systemic, 
political, economic and technological parameters. Also, Robinson's work (2009) develops the concept of 'information habitus' and finds that those (i.e. young people) with high-quality Internet access develop playful activities whereas those with low-quality access take a task-oriented stance in order to avoid "“wasteful" activities with no immediate payoff” (2009: 492). Robinson takes a particular perspective in studying a young population and draws conclusions about the role of the various qualities and disparities in life in the quality of Internet access and thus, in turn, in the quality of Internet use and skills. However, my study looks at the general population and goes directly beyond access, articulating a more concrete account of the role of everyday life and culture in Internet use, not just access. At the same time, the everyday context and its constraints are viewed here along policy parameters to explain Internet use divisions.

In broad terms, the findings reported in this article go beyond existing studies on ICT and Internet use, as they:

- move the discussion beyond binary divisions into 'haves' and 'have-nots', accounting for qualitative dimensions of the use of digital technologies such as the Internet;

- operationalise everyday life so as to brings together various everyday routines and practices (e.g. workplace, sociability, daily routines) and possible hints of resistance to technology within the everyday context (e.g. the Internet as a possible threat to traditions and values). This allows us to move beyond abstract or too-narrow accounts of the role of socio-cultural parameters in Internet use, translating such parameters in a tangible and sensible way;

- develop a bottom-up and top-down account of Internet use through the joint exploration of the role of people's everyday life and culture and their evaluation and awareness of top-down policies and regulations in the field. These two forces are positioned in a common framework where other parameters (e.g. demographics, access, etc) also operate.

In more theoretical terms, this article can add to the literature on ICTs some empirical insight into how the broad concepts of domestication, resistance culture and 'lifeword' can be empirically 
approached and operationalised in a quantitative study that tests the drivers of ICT use. Also, it moves beyond arguments which outline possible implications of aspects of everyday life for policy and regulation $[28 ; 13]$ and its results point to a deeper and more complex interaction between people's everyday life and their evaluation and awareness of policy and regulation as regards Internet use parameters.

Nevertheless, the discussion in this article has not gone deeper into exploring the direct interdependencies between everyday life and Internet policy and regulation in relation to Internet use. On the one hand, it has included only certain aspects of these two sets of factors in the analysis. On the other hand, it has not explored specifically the correlation between people's everyday life and their evaluation and awareness of Internet policy and regulation. The importance of everyday life for how Internet policies and regulations are evaluated is reported in a publication that constitutes part of the same study [31], but the close links of people's everyday life with their evaluation and awareness of policies and regulations in the field, and their role in Internet use, can also be examined through qualitative research. Qualitative examination can say more about how (and whether) aspects of everyday life interplay with policy and regulation, the qualitative aspects of this interplay and its implications for Internet use (e.g. policies and regulations can be seen as problematic by users living in particular everyday life contexts).

In any case, accounts of the role of people's everyday life and their evaluation and awareness of policy and regulation in Internet use, such as that reported in the present article, are particularly scant - not to say absent - in the literature, and more work is needed. 


\section{Notes}

${ }^{\text {i }}$ See, for instance: $32 ; 33 ; 34 ; 35 ; 36 ; 37 ; 6$.

ii See the introductory discussion of digital divides at the beginning of the article.

iii Cronbach's Alpha was higher than 0.7. This suggests that the constructs exhibit internal consistency and that each construct can be treated as a single indicator.

iv The variables looking at Internet policy and regulation focus on privacy and security because these are two issues of strong interest to ordinary people, compared to other areas of Internet policy and regulation.

v The demographic of income was not included as more than 60 per cent of the respondents declined to reveal their income.

vi Only three age groups were used in the analysis (15-24, 25-39 and 40-64). The age bracket 65+ was excluded to avoid multicollinearity and was used as a reference category.

vii For the regressions following 'Internet use', the everyday life and policy/regulation predictors were those included in the 'Internet use' regression (but they counted only answers given by Internet users) as well as the particular everyday life and policy questions asked to users only (e.g. evaluation of Internet policy and regulation (users); awareness of Internet authorities (users); everyday life and the Internet (users)) 


\section{References}

[1] Haddon L. Uneven patterns of adoption and use of ICTs. In: Information and Communication Technologies in Everyday Life: A Concise Introduction and Research Guide, Oxford: Berg; 2004, p. 18-43.

[2] Gunkel DJ. Second Thoughts: Toward a Critique of the Digital Divide. New Media and Society $2003 ; 5(4): 499-522$.

[3] Brady M. The Digital Divide Myth. E-commerce Times [online], 2000, URL (consulted Jun. 2006): http://www.ecomercetimes.com/story/3953.html

[4] Chaney H. The US “digital divide” is not even a virtual reality. Bridge News, 12 March 2000.

[5] Warschauer M. Technology and social inclusion: rethinking the digital divide. Cambridge, Massachusetts: MIT Press; 2003.

[6] Wyatt S, Thomas G, Terranova T. 'They came, they surfed and then went back to the beach': Conceptualizating use and non-use of the internet. In: Woolgar S, editor. Virtual Society? Technology, Cyberbole, Reality, Oxford: Oxford University Press; 2002, p. 23-40.

[7] Selwyn N. Reconsidering Political and Popular Understandings of the Digital Divide. New Media \& Society 2004; 6(3): 341-62.

[8] Hacker K, van Dijk J. The Digital Divide as a Complex and Dynamic Phenomenon. The Information Society $2003 ; 19(4): 315-26$.

[9] Perri 6 with Jupp B. Divided by Information? The "Digital Divide" and the Implications of the New Meritocracy. London: Demos; 2001.

[10] Katz J, Rice R. Social consequences of the Internet: Access, Involvement and Interaction. Cambridge, Mass: MIT Press; 2002.

[11] Robinson LA Taste for the Necessary. Information, Communication \& Society 2009; 12(4): 488-507.

[12] Baron SN, af Segerstad YH. Cross-cultural patterns in mobile-phone use: public space and 
reachability in Sweden, the USA and Japan. New Media \& Society 2010; 12(1): 13-34.

[13] Silverstone R. Media and Technology in the Everyday Life of European Societies. EMTEL 2000-2003; 2003.

[14] Silverstone R. Television and Everyday Life. London: Routledge; 1994.

[15] Silverstone R, Haddon L. Design and the Domestication of Information and Communication Technologies: Technical Change and Everyday Life. In: Silverstone R, Mansell R, editors. Communication by Design: the Politics of Information and Communication Technologies, Oxford: Oxford University Press. p. 44-74

[16] Silverstone R, Hirsch E, editors. Consuming Technologies: Media and Information in Domestic Spaces. London: Routledge; 1992.

[17] Silverstone R. Future Imperfect: Information and Communication Technologies in Everyday Life. In: Dutton W, editor. Information and Communication Technologies: Visions and Realities, Oxford: Oxford University Press; 1996, p. 217-32.

[18] Schutz A, Luckmann T. The Structures of the Life-world. London: Heinemann; 1974.

[19] Mokyr J. Technological inertia in economic history. Journal of Economic History 1992; 52(2): $325-38$.

20] Breakwell GM, Fife-Schaw C. Young people's attitudes toward new technology: Source and structure. New Directions for Child and Adolescent Development 1987; 35: 51-67.

[21] Hirschheim RA, Newman M. Information systems and user resistance. Theory and practice. The Computur Journal 1988; 31(5): 398-408.

[22] Northcott J, Fogerty M, Trevor M. Chips and Jobs: Acceptance of New Technology at Work. London- Policy Studies Institute; 1985.

[23] Willcocks L, Mason D. Computerising Work. People, Systems Design and Workplace Relations. London: Paradigm Press; 1987.

[24] Bauer M. editors. Resistance to New Technology - Nuclear Power, Information Technology, Biotechnology. Cambridge: Cambridge University Press; 1995a. 
[25] Bauer M. 'Technophobia': A Misleading Conception of Resistance to New Technology. In: Bauer M, editors. Resistance to New Technology, Cambridge: Cambridge University Press; 1995b, p. $97-122$

[26] Preston P. ICTs in Everyday Life: Public Policy Implications for 'Europe's Way to the Information Society. In Silverstone R, editors. Media, technology and everyday life in Europe: from information to communication, Ashgate: Aldershot; 2005, p. 195-211.

[27] Ricci A. Measuring information society. Dynamics of European data on usage of information and communication technologies in Europe since 1995. Telematics and Informatics 2000; 17(1/2): 141-67.

[28] Preston P. ICTs in Everyday Life: Public Policy Implications for 'Europe's Way to the Information Society. EMTEL 2000-2003; 2003.

[29] Frey JH. Survey Research by Telephone. $2^{\text {nd }}$ ed. Newbury Park, London, New Delhi: Sage Publications; 1989.

[30] Agresti A, Finlay B. Statistical Methods for the Social Sciences. $3^{\text {rd }}$ ed. N.J, London: Pearson Prentice Hall; 1997.

[31] NOT DISCLOSED. Role of social culture in evaluation of internet policies. The case of everyday and resistant culture in Greece. In: Adomi E, editor. Handbook of Research on Information Communication Technology Policy: Trends, Issues and Advancements, Hershey, USA: IGI Global; 2010, p. 631-51.

[32] Hartmann M. The Discourse of the Perfect Future - Young People and New Technologies. In: Silverstone R, editors. Media, technology and everyday life in Europe: from information to communication, Aldershot: Ashgate; 2005, p. 141-58.

[33] Horrigan J, Rainie L, Allen K, Madden M, O' Grady E. The Ever-Shifting Internet Population: A New Look of Internet Access and the Digital Divide. Washington, DC: Pew Internet and American Life Project; 2003.

[34] Kingsely P, Anderson T. Facing Life without the Internet. Internet Research: Electronic 
Networking Applications and Policy 1998; 8(4): 303-12.

[35] Mante-Meijer E, Haddon L, Concejero P, Klamer L, Heres J, Ling R, et al. Checking it out with the people. ICT markets and users in Europe. Heidelberg: Eurescom Project Report P903, Eurescom; 2001.

[36] Rommes E. “I Don't Know How to Fit it into my Life”: The Gap between Inclusion Initiatives and the Personal Stories of the Excluded. Paper presented at the EMTEL Conference New Media and Everyday Life in Europe, London, 23-26 April 2003.

[37] UK Online Centres. Understanding digital inclusion. A research summary. Sheffield: UK Online Centres; 2007.

Table 1: List of variables 


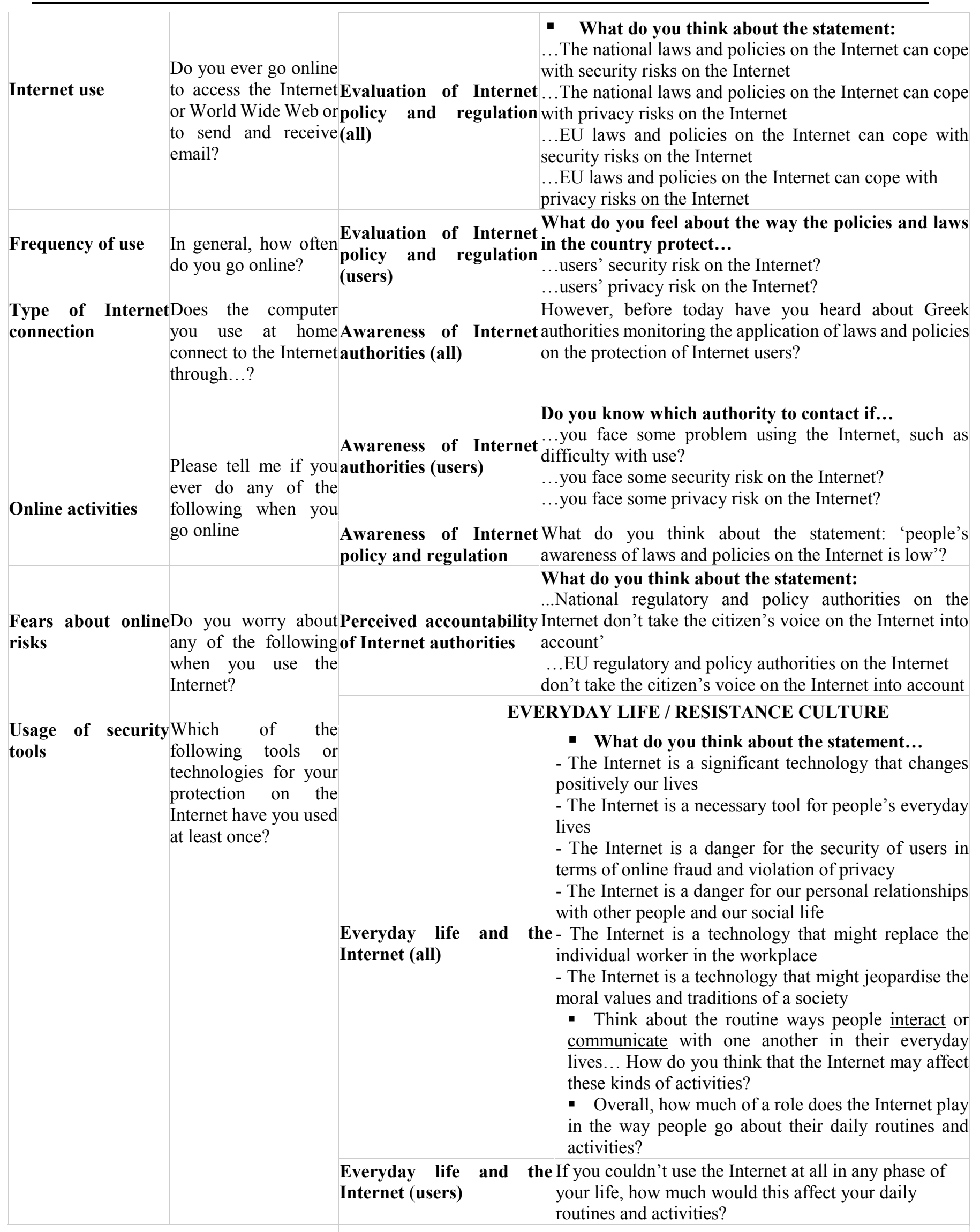

\section{DEMOGRAPHICS}




\section{Education}

Gender

Children in the household?

Media availability

Computer use

Frequency of watching TV

Frequency of reading newspaper History of Internet use
What is the last grade or class you completed at school?

What is your sex?

Do you have any children?

\section{ACCESS / OTHER MEDIA USE}

Does your household have...?

Do you ever use a computer at your workplace...or anywhere else?

How often do you match television programmes on average?

How often do you read newspapers on average? When did you first start going online?

Table 2: Internet use model

\begin{tabular}{|lcccccc|}
\hline \multicolumn{1}{|c}{ Variable } & B & S.E. & Wald & Df & Sig. & $\operatorname{Exp(B)}$ \\
\hline Age 15_24 & -2.077 & 0.556 & 13.927 & 1 & 0.000 & 0.125 \\
Age 25_39 & -1.012 & 0.468 & 4.682 & 1 & 0.030 & 0.364 \\
Education & -0.595 & 0.145 & 16.855 & 1 & 0.000 & 0.551 \\
Gender & 1.482 & 0.422 & 12.334 & 1 & 0.000 & 4.403 \\
Media availability: Internet & -3.247 & 0.434 & 55.978 & 1 & 0.000 & 0.039 \\
Computer use & 3.595 & 0.548 & 43.046 & 1 & 0.000 & 36.434 \\
Constant & -1.936 & 1.051 & 3.394 & 1 & 0.065 & 0.144 \\
Chi-Square & & & 340.264 & & \\
p value & & & 0.000 & & \\
Nagelkerke $\mathbf{R}^{2}$ & & & 0.789 & & \\
\hline
\end{tabular}

Base: $\mathrm{N}=1001$ (Internet users \& non-users)

Table 3: Frequency of use model

\begin{tabular}{|lcccccc|}
\hline & Variable & B & S.E. & Beta & T & Sig. \\
$($ Constant $)$ & & 3.860 & 0.544 & & 7.094 & 0.000
\end{tabular}




\begin{tabular}{|lrrrrr|} 
Children in the household & -0.019 & 0.007 & -0.186 & -2.776 & 0.006 \\
Media availability: Internet & -1.418 & 0.296 & -0.320 & -4.799 & 0.000 \\
Dial-up & 0.533 & 0.150 & 0.241 & 3.548 & 0.001 \\
History of use & -0.224 & 0.079 & -0.194 & -2.848 & 0.005 \\
Everyday life and the Internet (users) & -0.267 & 0.076 & -0.242 & -3.531 & 0.001 \\
Awareness of Internet authorities (users) & 0.399 & 0.175 & 0.158 & 2.283 & 0.024 \\
R-Squared & & 0.391 & & \\
F & & 15.299 & & \\
P value & & 0.000 & \\
\hline
\end{tabular}

Base: $\mathrm{N}=445$ (Internet users)

Table 4: Online activities model

\begin{tabular}{|lrrrrr|}
\hline \multicolumn{1}{c}{ Variable } & B & S.E. & Beta & T & Sig. \\
(Constant) & 2.272 & .351 & & 6.474 & .000 \\
Dial-up & -.748 & .222 & -.274 & -3.368 & .001 \\
Everyday life and the Internet (users) & .247 & .110 & .183 & 2.249 & .026 \\
R-Squared & & & 0.132 & & \\
F & & & & & \\
P value & & & 0.000 & \\
\end{tabular}

Base: $\mathrm{N}=445$ (Internet users)

Table 5: Dial-up model

\begin{tabular}{|lcccccc|}
\hline \multicolumn{1}{|c}{ Variable } & B & S.E. & Wald & Df & Sig. & $\operatorname{Exp(B)}$ \\
Frequency of use & 0.589 & 0.179 & 10.810 & 1 & 0.001 & 1.801 \\
Online activities & -0.483 & 0.155 & 9.682 & 1 & 0.002 & 0.617 \\
Constant & -0.158 & 0.565 & 0.078 & 1 & 0.779 & 0.854
\end{tabular}




\begin{tabular}{|lc|}
\hline Chi-Square & 28.922 \\
P value & 0.000 \\
Nagelkerke $\mathbf{R}^{2}$ & 0.235
\end{tabular}

Base: $\mathrm{N}=445$ (Internet users) 